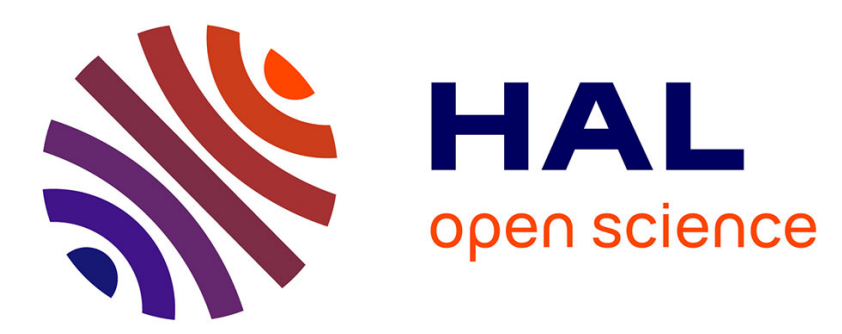

\title{
Rheological Investigation of Polybutadienes Having Different Microstructures over a Large Temperature Range
}

\author{
Reiner Zorn, Gregory B. Mckenna, Lutz Willner, Dieter Richter
}

\section{- To cite this version:}

Reiner Zorn, Gregory B. Mckenna, Lutz Willner, Dieter Richter. Rheological Investigation of Polybutadienes Having Different Microstructures over a Large Temperature Range. Macromolecules, 1995, 28 (25), pp.8552-8562. 10.1021/ma00129a014 . hal-02564337

\section{HAL Id: hal-02564337 \\ https://hal.science/hal-02564337}

Submitted on 5 May 2020

HAL is a multi-disciplinary open access archive for the deposit and dissemination of scientific research documents, whether they are published or not. The documents may come from teaching and research institutions in France or abroad, or from public or private research centers.
L'archive ouverte pluridisciplinaire $\mathbf{H A L}$, est destinée au dépôt et à la diffusion de documents scientifiques de niveau recherche, publiés ou non, émanant des établissements d'enseignement et de recherche français ou étrangers, des laboratoires publics ou privés. 


\title{
Rheological Investigation of Polybutadienes Having Different Microstructures over a Large Temperature Range
}

\author{
Reiner Zorn, ${ }^{*,+}$ Gregory B. McKenna, ${ }^{*, *}$ Lutz Willner, ${ }^{\dagger}$ and Dieter Richter ${ }^{\dagger}$ \\ Forschungszentrum Jülich, IFF, D-52425 Jülich, Germany, and National Institute of \\ Standards and Technology, Gaithersburg, Maryland 20899
}

Received February 22, 1995; Revised Manuscript Received July 24, 1995

\begin{abstract}
The rheological behavior of five polybutadienes having different microstructures has been characterized using dynamic mechanical, stress relaxation, and viscosity measurements in a temperature range from just below the conventional glass transition to $100^{\circ} \mathrm{C}$ above it. The data covered a broad enough frequency (time) and temperature range that we were able to characterize the responses in both the glassy and terminal dispersions of the polymers and to address the question of the validity of thermorheological simplicity. Uncritical application of time-temperature superposition principles to these data resulted in reduced viscoelastic responses that cover 12-14 decades in frequency or time. Close examination of the data in the glassy and terminal dispersions shows that the temperature shift factors required to superpose the data in the two regions are, however, different. Such a deviation from thermorheological simplicity can be analyzed within the framework of the coupling model of $\mathrm{Ngai}^{1-3}$ that relates the shapes of the dispersion to the temperature dependence of the viscoelastic spectrum. Comparison of the polybutadienes shows differences in glass transition temperature, shape of the segmental relaxation, and fragility that depend on microstructure. Increasing the content of vinyl side groups causes an increase of spectral broadening as well as an increase in fragility-two features which can be related within the coupling model.
\end{abstract}

\section{Introduction}

Polybutadienes with different microstructures can be synthesized by anionic polymerization. ${ }^{4}$ When the conditions of the synthesis (cosolvent and temperature) are changed, the vinyl content can be controlled over the range $0.07-0.98$ while the cis/trans ratio remains nearly constant at about 0.7 . Hence, these polymers offer the opportunity to study the influence of an increasing number of short, rigid side groups on an otherwise unchanged polymer.

It is well-known that the glass transition of polybutadienes varies as a function of vinyl content. As a consequence, many macroscopic features (e.g. the abrasion resistance ${ }^{5}$ ) depend strongly on this parameter. To the extent that such properties depend on the rheological behavior of the polymer, these might be expected to vary with molecular architecture-raising the question as to whether the viscoelastic response can be explained simply as a shift following the offset of $T_{\mathrm{g}}$ or if a more complicated dependence is observed.

The viscoelastic properties (e.g. storage and loss modulus) as a function of temperature, frequency, or time can be divided roughly into four regions: 6 terminal, plateau, transition, and glassy zone (in the order from high to low temperature or low to high frequency). The first three zones can be described at least qualitatively by theories which build on the concept of the polymer as a connection of many monomers (Rouse model, reptation, etc.). On the other hand, adequate descriptions of the behavior in the glassy zone are currently unattainable, perhaps due to the dependence in the glass on local mobility rather than the connectivity of the polymer molecule. While the rheological properties of 1,2-1,4-polybutadienes have been studied previously in the terminal and plateau zones ${ }^{7}$ and in the glassy $z^{2}{ }^{8}{ }^{8}$ the investigations were performed on different

\footnotetext{
† Forschungszentrum Jülich.

$\ddagger$ NIST

Abstract published in Advance ACS Abstracts, November 15,
}

samples and therefore they are not readily comparable. Furthermore, in the latter case, ${ }^{8}$ the polymers were lightly cross-linked. In this work for the first time the full range of rheological behavior is investigated on the same series of samples. Additionally, in a future publication the dielectric response of the same series of polybutadienes will be reported.

In this investigation we explore the validity of the concept of thermorheological simplicity (time-temperature superposition). When time-temperature superposition is valid, the entire time (frequency)-temperature range studied is describable using a single temperature dependence of the time scale, i.e. a single temperature shift factor $a_{T}$. While the validity of this concept is widely accepted, at least as a reasonable approximation to material behavior (cf. ref 6), there have been renewed efforts recently to examine apparent violations more closely. ${ }^{1-3,8,9}$ One explanation for deviations from thermorheologically simple behavior has been offered in the coupling model of Ngai. ${ }^{1-3}$ Here one starts from a unique fundamental shift factor $a_{T}{ }^{0}$ on the microscopic level. Then it is postulated that the glassy dispersion and the terminal dispersion are "coupled" differently to their complex environment, and hence shift differently with temperature. The degree of coupling is determined empirically from the shape of the appropriate relaxation spectrum and the resulting two coupling parameters $n_{\alpha}$ and $n_{\eta}$ can be used to calculate the temperature dependence of the shift factor, as described subsequently. Here we note that $n_{\alpha}$ refers to the segmental relaxation which governs the glassy zone and $n_{\eta}$ that for the terminal relaxation. A difference $\left(n_{\alpha} \neq n_{\eta}\right)$ gives rise to the deviations from thermorheological simplicity because the glassy and terminal relaxations shift differently with temperature.

Although the coupling parameters cannot be derived quantitatively from the molecular structure of the polymer, they can be determined independently from the breadth (shape) of the relaxation spectrum. If timedependent properties, such as the modulus $G(t)$ or the compliance $J(t)$, can be described by the Kohlrausch- 
Williams-Watts (KWW) stretched exponential function $\left\{\exp \left(-(t / \tau)^{\beta}\right)\right\}$ the coupling parameter is simply related by $n=1-\beta$. Correspondingly, an important test of this model is the comparison of the relaxation spectrum shape, i.e., $n_{\alpha}$ and $n_{\eta}$ with the temperature dependence of time scale, i.e., $a_{T}$. Recently, a more extensive application of the coupling model ${ }^{9}$ suggests that the fundamental shift factor $a_{T}^{0}$ has the same temperature dependence relative to the glass transition temperature for the polymers studied. Such a possible universality of behaviors makes the coupling model worth exploring further.

\section{Experimental Methods}

2.1. Materials. Five polybutadienes with different microstructures were prepared by anionic polymerization using high-vacuum and break-seal techniques, following the procedures outlined by Morton and Fetters. ${ }^{4} s$-Butyllithium purchased from Fluka Chemie $\mathrm{AG}^{32}$ in a $1.3 \mathrm{M}$ solution in cyclohexane was used as initiator. For purification the solvent was evaporated and the residual organolithium was distilled onto a cold finger. The pure initiator was dissolved in $n$-hexane and split into ampules fitted with break-seals. With one of the ampules a test polymerization of styrene was carried out. The content of organolithium was calculated from the molecular weight of the resulting polystyrene determined by size exclusion chromatography (SEC).

1,3-Butadiene was obtained by thermal decomposition of 3-sulfolene (Aldrich-Chemie-GmbH) ${ }^{32}$ at $103^{\circ} \mathrm{C}$. In order to remove sulfur dioxide the gaseous decomposition products were guided twice through $20 \%$ potassium hydroxide solution and over potassium hydroxide pellets. The butadiene gas was collected, purified with n-butyllithium at $-78^{\circ} \mathrm{C}$, and freshly distilled into the reaction vessel. A mixture of cyclohexane and benzene (25 vol \%) was used as polymerization solvent. In order to generate polybutadienes with increased vinyl content, tetrahydrofuran (THF), diethyl ether (DEE) (both Merck p.A. grade), ${ }^{32}$ and dipiperidinoethane (DIPIP) (Sigma Chemie GmbII $)^{32}$ were added as polar modifiers. Cyclohexane and benzene were purified with $n$-butyllithium, THF, and DEE with sodium potassium alloy/ benzophenone. DIPIP was treated with $\mathrm{CaH}_{2}$ for 2 days and then distilled under high vacuum (bp $55^{\circ} \mathrm{C} / 3 \mathrm{~Pa}$ ) into break-seal ampules.

The polymerizations were performed in sealed reactors at an active center concentration of about $4 \times 10^{-3}$ $\mathrm{mol} / \mathrm{L}$. In order to obtain a polymer with a high level of 1,4-addition the polymerization was carried out in a cyclohexane/benzene mixture in the absence of a modifier. For intermediate microstructures DEE was used as cosolvent in a molar ratio of $r \approx 1000$. The polymerizations were conducted for $22 \mathrm{~h}$ at $0^{\circ} \mathrm{C}$ and for $5 \mathrm{~h}$ at $30^{\circ} \mathrm{C}$, respectively. Polymers with high vinyl contents were obtained using THF $(r=22)$ and DIPIP $(r=12)$ as modifiers. In both cases the duration of the reaction was $1 \mathrm{~h}$. Termination of all polymerizations was done by addition of degassed methanol. The polymers were isolated by precipitation in methanol and dried under high vacuum to a constant weight.

The polybutadienes were analyzed by SEC using a Waters 150-C ALC/GPC system. ${ }^{32}$ The measurements were performed at $30^{\circ} \mathrm{C}$ on an Ultrastyragel column system (linear, $500,10^{3}, 10^{4}, 10^{5} \AA$ nominal pore size) with THF as carrier solvent at a flow rate of $1 \mathrm{~mL} / \mathrm{min}$. The chromatograms reveal that all samples possess near monodisperse molecular weight distributions with poly-
Table 1. Molecular Weights, Microstmucture Mole Fraction, and Glass Transition Temperatures of Anionically Polymerized Polybutadienes Used in This Investigation

\begin{tabular}{lcccccc}
\hline sample & $M_{\mathrm{n}}{ }^{a}$ & $1,2^{b}$ & 1,4-trans $^{b}$ & 1,4-cis $^{b}$ & cosolvent & $\begin{array}{c}T_{\mathrm{g}} \\
\left.{ }^{\circ} \mathrm{C}\right)\end{array}$ \\
\hline PB 7 & 21 & $0.07(0.07)$ & 0.52 & 0.41 & & -95 \\
PB 53 & 18 & $0.52(0.53)$ & 0.29 & 0.19 & DEE $^{c}$ & -57 \\
PB 70 & 20 & $0.68(0.70)$ & 0.20 & 0.12 & DEE $^{d}$ & -35 \\
PB 86 & 20 & $0.86(0.86)$ & 0.08 & 0.06 & THF $^{-3}$ & -23 \\
PB 95 & 20 & $0.95(0.95)$ & $e$ & & DIPIP & -1
\end{tabular}

a By membrane osmometry in toluene at $37^{\circ} \mathrm{C} .{ }^{b}$ Mole fraction by ${ }^{13} \mathrm{C}$ NMR, values in parentheses by ${ }^{1} \mathrm{H}$ NMR. ${ }^{c}$ Polymerization temperature $30^{\circ} \mathrm{C}$. ${ }^{d}$ Polymerization temperature $0^{\circ} \mathrm{C} .{ }^{e} 1,4$-cis + 1,4-trans: 0.05

dispersity indices $\left(M_{\mathrm{w}} / M_{\mathrm{n}}\right)$ smaller than 1.05 . The number-average molecular weights $M_{\mathrm{n}}$ were determined with a Knaur membrane osmometer ${ }^{32}$ at $37^{\circ} \mathrm{C}$. The solvent was toluene distilled over $\mathrm{CaH}_{2}$. The $M_{\mathrm{n}}$ values were obtained from plots of $(\Pi / c)^{1 / 2}$ vs $c$ where $\Pi$ is the osmotic pressure and $c$ is the concentration of polymer. In all cases the correlation coefficient of the linear regression was better than 0.99 .

The microstructural composition of the polybutadienes was determined by ${ }^{1} \mathrm{H}$ and ${ }^{13} \mathrm{C}$ NMR spectroscopy in deuteriochloroform at room temperature. The spectra were recorded on a Bruker WM300 spectrometer ${ }^{32}$ at 300.13 and $75.47 \mathrm{MHz}$, respectively. The ${ }^{13} \mathrm{C}$ measurements were performed using ${ }^{1} \mathrm{H}$ broadband decoupling techniques with suppression of the Nuclear Overhauser enhancement. A pulse delay of $5 \mathrm{~s}$ was used. It was shown by van der Velden et al. ${ }^{10}$ and Sato et al.11 that this time is sufficiently long to obtain spectra for a quantitative evaluation. The ${ }^{1} \mathrm{H}$ NMR spectra were analyzed in order to determine the vinyl content of the polybutadienes. For this purpose the relative integrals of the broad olefin proton bands around 4.9 and $5.4 \mathrm{ppm}$ were used. Additionally, the percentage of the vinyl units was obtained from ${ }^{13} \mathrm{C}$ NMR spectra using the resonances of the unsaturated carbons between 114 and $143 \mathrm{ppm}$. The results of both techniques are in good agreement. They are included in Table 1 in parentheses.

For the determination of the cis/trans ratio the aliphatic region of the ${ }^{13} \mathrm{C}$ spectrum was used. It is known that signals at 24.9 and $27.4 \mathrm{ppm}$ are due to the $\mathrm{CH}_{2}$ group of 1,4-units in cis-vinyl and those at 30.2 and $32.7 \mathrm{ppm}$ to trans-vinyl and trans-1,4 sequences, respectively. ${ }^{12,13}$ Taking into account a random distribution of sequences, the relative peak areas are representative for this cis/tans ratio of the polybutadienes. The results obtained are shown in Table 1 .

It should be noted that under the reaction conditions used, a conversion close to $100 \%$ vinyl units was expected with the use of DIPIP as polar modifier. ${ }^{14,15}$ However, ${ }^{13} \mathrm{C}$ and ${ }^{1} \mathrm{H}$ NMR clearly indicate $5 \% 1,4-$ content. Possibly, this is due to polar impurities present in the commercial DIPIP which could not be removed by the purification process. It is known that DIPIP can be easily replaced from the growing chain end by other polar modifiers, thereby reducing the 1,2-content of the resulting polybutadiene. 15

The glass transition temperatures $T_{\mathrm{g}}$ were obtained by modulated DSC using a TA Instruments Thermal Analyst 2200 differential scanning calorimeter. ${ }^{32}$ The heating rate was set to $5 \mathrm{~K} / \mathrm{min}$ with a modulation frequency of $1 \mathrm{~min}^{-1}$ and an amplitude of $0.5 \mathrm{~K}$. The observed widths of the glass transition were in the range 


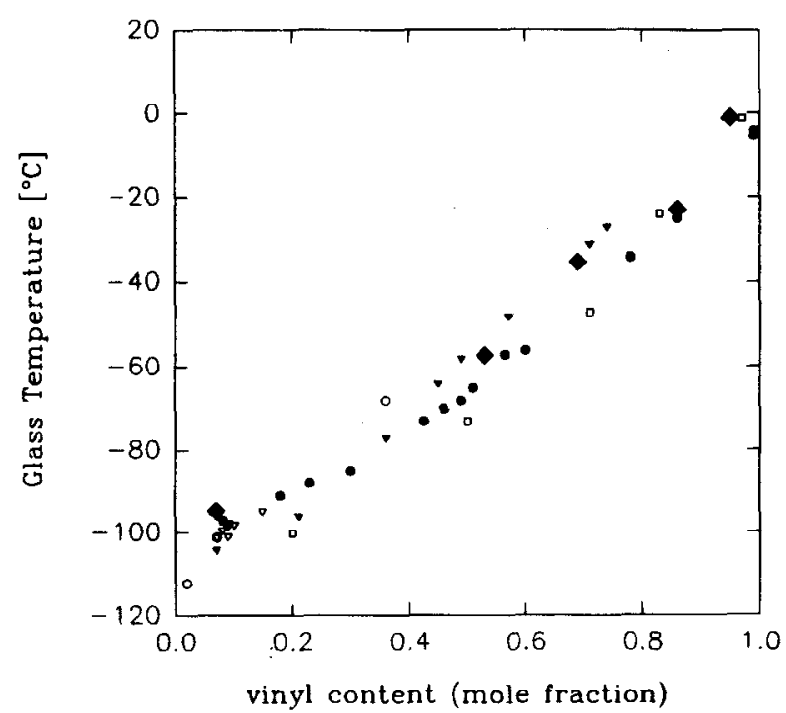

Figure 1. Glass transition temperature of cis/trans-vinylpolybutadienes for various microstructures. Our data: Literature data: $(0)$ ref $6 ;(0)$ ref $7 ;(\nabla)$ ref 16 (data with $M_{\mathrm{w}}$ $>20000$ only); ( $\nabla$ ) ref 17 ; ( $\square$ ) ref 8 .

of 5-9 K; the points of inflection of the reversible heat flow are listed in Table 1 as $T_{\mathrm{g}}$. Figure 1 shows our values for $T_{\mathrm{g}}$ compared with those from the literature ${ }^{6-8,16,17}$ plotted against the vinyl content of the polybutadienes. It can be seen that our values are compatible with the general $T_{\mathrm{g}}\left(x_{\text {vinyl }}\right)$ relationship but also that the combined uncertainties due to the calorimetric determination of $T_{\mathrm{g}}$, microstructure characterization, and differences in values obtained by different laboratories lead to a considerable scatter in the plot on the order of $\pm 5 \mathrm{~K}$. Nevertheless, a simple linear relation as given by Bahary et al. ${ }^{5}$ based on the theory of the glass transition of Gibbs and Di Marzio ${ }^{18}$ appears unlikely. Figure 1 evidences a deviation from this linearity with an increasing dependence of $T_{\mathrm{g}}$ on $x_{\mathrm{vinyl}}$ as $x_{\text {vinyl }}$ increases.

2.2. Rheological Techniques. In order to cover the large temperature range studied here three different techniques had to be used. Nevertheless, all measurements were performed on the same rotary rheometer (Rheometrics RMS-800) ${ }^{32}$ without removing the sample between the experiments or changing its geometry.

Obtaining good temperature control turned out to be a major problem for temperatures below $-20{ }^{\circ} \mathrm{C}$. Although the rheometer has a cooling system using liquid nitrogen which is able to attain temperatures below $-120^{\circ} \mathrm{C}$, ice builds up on the moving parts, eventually blocking them. The solution that we used was to enclose the oven and the moving parts in a plastic bag. The evaporating nitrogen displaces the (possibly humid) air from the inside of the bag and ice condensation is reduced significantly. The effectiveness of this method was further enhanced by putting dishes with desiccant inside the bag.

Because the built-in temperature sensor of the rheometer does not measure at the sample position, there is a considerable difference between the temperature given by the instrument and the true sample temperature. Therefore the latter was calibrated against a thermocouple (which was itself calibrated against NIST standards) inserted into the sample after the rheological experiments were finished. The calibration revealed deviations of -0.7 to $+1.8 \mathrm{~K}$ in the temperature range -100 to $+50^{\circ} \mathrm{C}$. Here we report the temperatures as determined from the calibration procedure.
In all experiments a parallel-plate geometry was used. (The samples were too viscous at room temperature to be molded into a cone-and-plate arrangement on the rheometer.) The diameter of the cylindrical samples was $8 \mathrm{~mm}$, their thicknesses were $1.0-2.3 \mathrm{~mm}$.

Dynamic Measurements. Storage and loss moduli $G^{\prime}(\omega)$ and $G^{\prime \prime}(\omega)$, were measured as functions of frequency in the range $0.01 \mathrm{rad} / \mathrm{s}<\omega<100 \mathrm{rad} / \mathrm{s}$. Subsequently, these functions were rescaled to master curves using horizontal and vertical shift factors, $a_{T}$ and $b_{T}$, respectively:

$$
\begin{aligned}
& G^{\prime}(\omega, T)=b_{T} G^{\prime}\left(a_{T} \omega, T_{0}\right) \\
& G^{\prime \prime}(\omega, T)=b_{T} G^{\prime \prime}\left(a_{T} \omega, T_{0}\right)
\end{aligned}
$$

The rescaling was performed with an automatic computer program (LSSHIFT) using a least-squares criterion to optimize the quality of the master curve. ${ }^{20}$

By application of different strain values $(0.5-150 \%)$ the dynamic response could be effectively determined when the modulus of the sample was between $10^{1}$ and $10^{9} \mathrm{~Pa}$. [Note that dynamic measurements at different strain settings and at appropriate temperatures were performed to establish that the moduli were independent of strain.] The temperature range in which we could characterize $G^{\prime}$ and $G^{\prime \prime}$ for the polybutadienes of fixed geometry was therefore about $T_{\mathrm{g}}-5 \mathrm{~K}$ to $T_{\mathrm{g}}+70$ $K$ with respect to the individual glass transition temperatures $T_{\mathrm{g}}$. For one of the samples (PB 7) we found an anomalous increase of the moduli in the temperature range -54 to $-76^{\circ} \mathrm{C}$. The modulus increased progressively with measurement time. After ruling out all possibilities of instrumental artifacts, we can explain this phenomenon only by (maybe shear-induced) crystallization. The shift factors from this region have been omitted from our analysis.

Viscosity Measurements. For higher temperatures the moduli at the low-frequency end were no longer able to be reliably measured. Furthermore, here the knee between Newtonian behavior and the rubbery plateau shifts out of the frequency window so that $a_{T}$ and $b_{T}$ could not be determined independently. In order to obtain information in this range the rheometer was used as a viscometer in "steady" mode. A constant strain rate was applied, and the viscosity was calculated from the resulting torque once steady state was attained. The strain rate was chosen large enough to obtain a reliable value for the torque but significantly smaller than the onset of nonlinearity.

The temperature dependence of the zero shear rate viscosity involves both shift factors, which follows from (1) and (2):

$$
\eta_{0}(T)=a_{T} b_{T} \eta_{0}\left(T_{0}\right)
$$

In order to extend the temperature range at which we could determine the shift factor $a_{T}$, we assumed the vertical shift factor $b_{T}$ to be constant in the hightemperature range. This is reasonable on the basis of an extrapolation of the results from the dynamic modulus measurements (Figure 3 discussed subsequently). The lowest viscosity accessible with the instrument at the given sample geometry was about $100 \mathrm{~Pa} \mathrm{~s}$, which made experiments up to about $T_{\mathrm{g}}+120 \mathrm{~K}$ possible.

Stress Relaxation Measurements. At the lowest temperatures, dynamic mechanical measurements could not be performed because the samples broke from the platens. Similarly, step strain experiments in the 
"dynamic" mode of the instrument could not be performed since the minimum strains applied to the samples were still large enough to cause failure at the platen-sample interface. Therefore, we performed stress relaxation experiments in the "steady" mode of the rheometer, which allowed us to apply very small strains and keep the torques low enough that failure did not occur. A constant strain rate pulse of $2 \mathrm{~s}$ duration was applied, resulting in a strain of $0.1 \%$. Then, the decay of the stress was registered over 400$10000 \mathrm{~s}$. The ratio stress/strain gives the stress relaxation modulus $G(t)$ which is the inverse Fourier transform of the complex dynamic modulus $G^{*}(\omega)=G^{\prime}(\omega)+$ $\mathrm{i} G^{\prime}(\omega)$. Assuming the validity of the same timetemperature superposition property as above, a master curve was constructed by

$$
G(t, T)=b_{T} G\left(t / a_{T}, T_{0}\right)
$$

using the same computational procedure as for the dynamical moduli.

For two reasons, this technique allowed us to extend our accessible temperature range: First, smaller strains could be applied using relaxation after a constant shear rate method described above than in the normal "dynamic" mode of the instrument. Hence, we could work at temperatures below the DSC-measured $T_{\mathrm{g}}$. Secondly, the long time range of the experiments includes lowfrequency information down to $10^{-4} \mathrm{~s}^{-1}$ which cannot be accessed in the dynamic mode without running the experiment for days.

The lowest temperatures where this method could be used were about $\mathrm{T}_{\mathrm{g}}-10 \mathrm{~K}$. Below this the samples tended to detach from the platens, leading to abrupt drops in the $G(t)$ curves. The gain of $5 \mathrm{~K}$ by using this method seems small but it has to be noted that the range of the horizontal shift factor $a_{T}$ is extended by two or three decades because of its strong temperature dependence below $T_{\mathrm{g}}$.

\section{Results and Discussion}

3.1. Dynamic Mechanical Measurements. Figure 2 shows the dynamic moduli $G^{\prime}(\omega)$ and $G^{\prime \prime}(\omega)$ for the sample PB 70 which has $68 \% 1,2$ content. The data are for 29 temperatures in the range -43.1 to $+25.3^{\circ} \mathrm{C}$. Figure 3 shows the master curve that results from performing time-temperature shifting of these curves. Figure 4 shows the shift factors from the reduction procedure. A large deviation from the time-temperature reduction occurs in the $G^{\prime \prime}(\omega)$ reduced data for high frequencies at the lowest temperatures $\left(T<T_{\mathrm{g}}-5 \mathrm{~K}\right)$. This is obviously due to a systematic error of the measurement at those extreme conditions since the upturn in $G^{\prime \prime}(\omega)$ is not accompanied by an equivalent feature in $G^{\prime}(\omega)$, thus violating the Kramers-Kronig relationship. More interesting are the minor deviations of both $G^{\prime}$ and $G^{\prime \prime}$ data from time-temperautre superposability in the regions of large curvature which are visible for all samples. These could indicate an invalidity of thermorehological simplicity due to different shift factors for local segmental relaxations and terminal relaxations. This kind of anomaly has been found previously in polystyrene and other polymers ${ }^{21}$ and was subsequently rationalized within the framework of the coupling model of Ngai. ${ }^{1-3}$

We have examined the polybutadiene data reported here for such a lack of thermorheological simplicity by splitting the moduli curves at a transition frequency
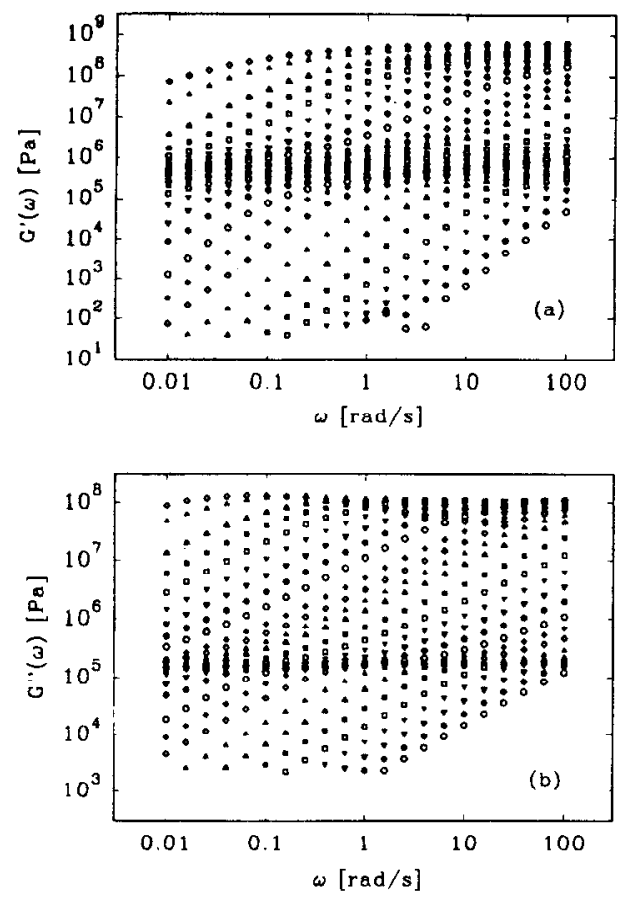

Figure 2. Dynamic moduli for polybutadiene sample PB 70: (a) storage modulus and (b) loss modulus vs angular frequency. Test temperatures in ${ }^{\circ} \mathrm{C}$ : (O) $25.3 ;(0) 21.6 ;(\nabla) 16.7 ;(\nabla) 11.9$;

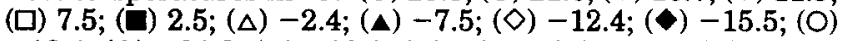
$-18.4 ;(\circlearrowleft)-21.3 ;(\nabla)-22.9 ;(\nabla)-25.1 ;(\square)-27.2 ;(\square)-29.2$; $(\Delta)-31.1 ;(\Delta)-32.1 ;(\diamond)-33.0 ;(\diamond)-33.9 ;(0)-35.0 ;(\bullet)$ $-36.1 ;(\nabla)-37.2 ;(\nabla)-38.2 ;(\square)-39.1 ;(\square)-40.0 ;(\Delta)-40.9$; (৯) $-41.8 ;(\diamond)-43.1$.

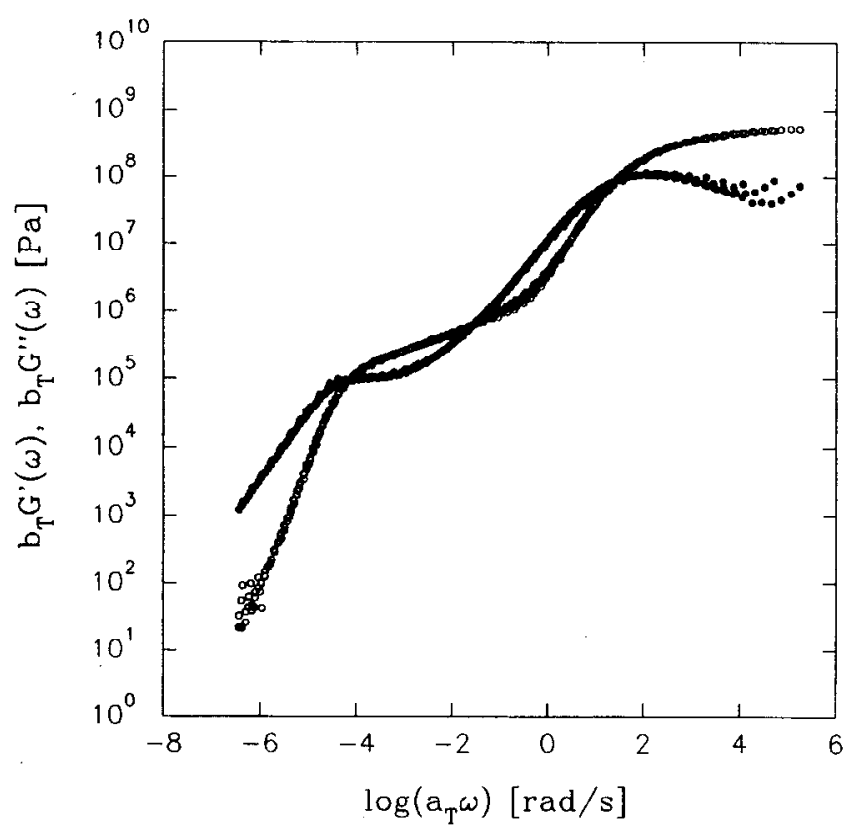

Figure 3. Master curve of the dynamic moduli for polybutadiene sample PB 70 constructed from the data of Figure 2: (O) $G^{\prime}(\omega)$; (O) $G^{\prime \prime}(\omega)$.

(inflection point, e.g., for PB $70, \log _{10}\left(a_{T} \omega_{\text {split }}\right)=-2.2$; see Figure 5) and rescaling the parts separately. Although there is a considerable overlap between segmental and terminal relaxation the high-frequency parts which are dominated by segmental relaxation should scale slightly differently from the low-frequency parts which are mainly due to the terminal relaxation process. Indeed, the master curve construction shows that there is a significant difference between the shift factors of segmental and terminal relaxation. The ratio $\log _{10} a_{T, \alpha}$ ! 

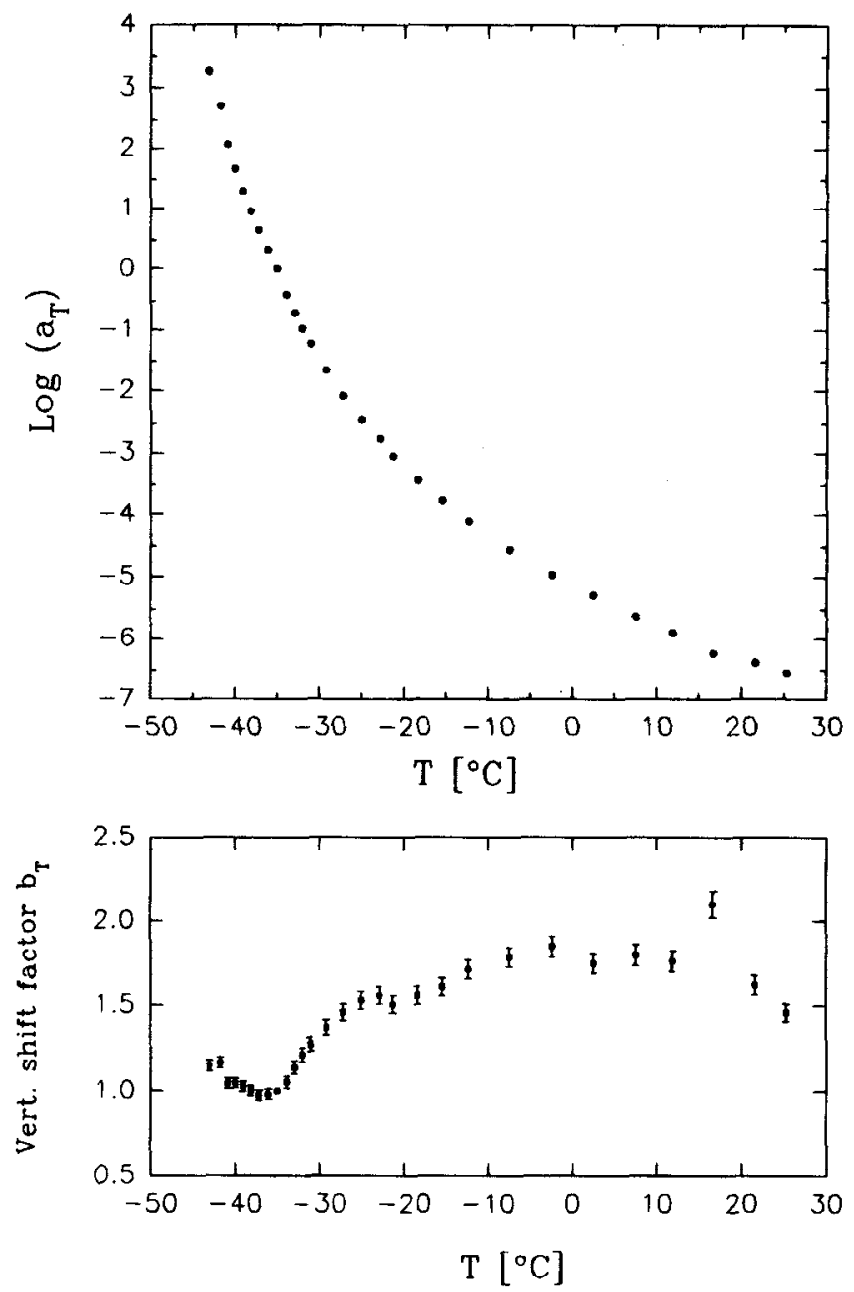

Figure 4. Shift factors obtained from the construction of Figure 3. The reference temperature is $T_{0}=-35^{\circ} \mathrm{C}=T_{\mathrm{g}}$. For the vertical shift factor (statistical) errors derived from the master curve construction are shown; in the case of the horizontal shift factors those errors are smaller than the symbol size.

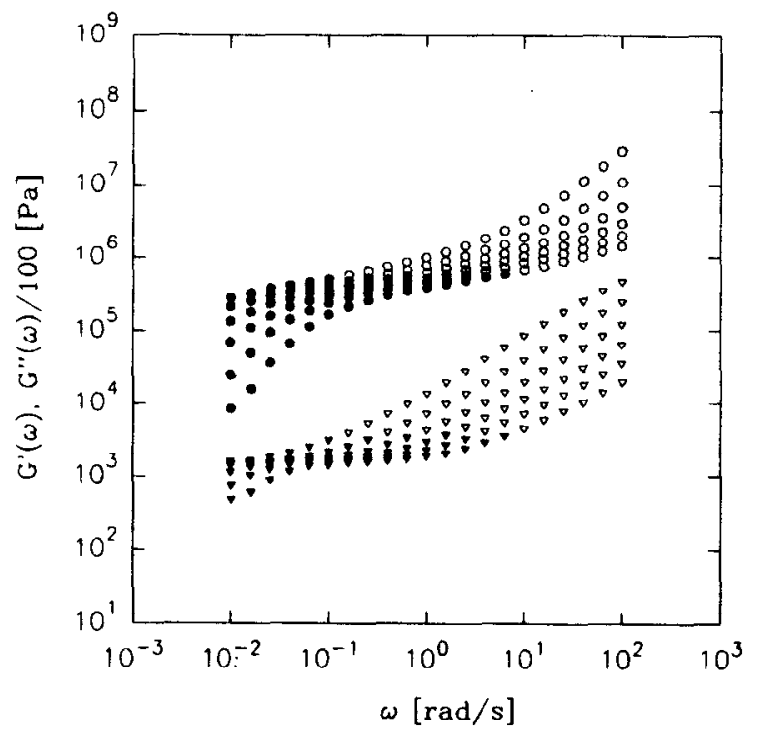

Figure 5. Illustration of the splitting used to make obvious deviations from thermorheological simplicity. The parts shown as hollow circles have been related to the segmental relaxation which yields the shift factor $a_{T, \alpha}$. The full circles are for the terminal relaxation which yields $a_{T, \eta}$.

$\log _{10} a_{T, \eta}$ is always $>1$ (except for two outliers), showing that the temperature shift for the segmental relaxation

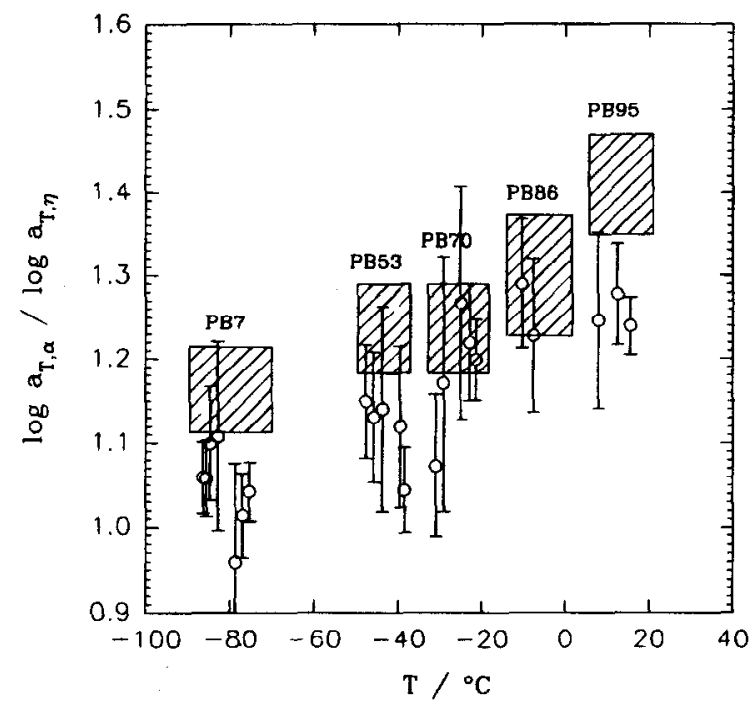

Figure 6. Plot of the ratio $\log _{10} a_{T, 0} / \log _{10} a_{T, \eta}$ for all five polybutadiene samples vs temperature. The hatched areas show the values expected from the coupling model with $\beta_{\eta}=$ $0.575 \pm 0.025$ assumed.

Table 2. Comparison of the Proportionality Factor $\boldsymbol{R}$ between the Segmental and Terminal Shift Factors with the Value Predicted from the Coupling Model Using (*) the Range of $\beta$, Reported in the Literature and (**) the Arbitrarily Chosen Value $\beta_{\eta}=0.515^{a}$

\begin{tabular}{ccccc}
\hline sample & $\beta_{\alpha}$ & $\mathrm{R}$ & \multicolumn{1}{c}{$\beta_{\eta}{ }^{*} / \beta_{\alpha}$} & $\beta_{\eta}{ }^{* * / \beta} \beta_{\alpha}$ \\
\hline PB 7 & $0.494[0.004]$ & $1.049[0.011]$ & $1.164[0.052]$ & $1.043[0.008]$ \\
PB 53 & $0.466[0.001]$ & $1.099[0.023]$ & $1.235[0.054]$ & $1.105[0.002]$ \\
PB 70 & $0.466[0.001]$ & $1.186[0.027]$ & $1.235[0.054]$ & $1.105[0.002]$ \\
PB 86 & $0.443[0.016]$ & $1.044[0.145]$ & $1.298[0.073]$ & $1.163[0.042]$ \\
PB 95 & $0.408[0.002]$ & $1.248[0.011]$ & $1.409[0.062]$ & $1.262[0.006]$
\end{tabular}

a Note that the numbers in brackets are estimates of the asymptotic standard errors of the parameters as determined from the nonlinear curve-fitting procedure (see ref 34 ).

is stronger than that for the terminal relaxation.

According to Ngai's ${ }^{1-3}$ coupling model, this ratio should be equal to the ratio of the stretching exponents for terminal and segmental relaxations:

$$
R=\frac{\log _{10} a_{T, \alpha}}{\log _{10} a_{T, \eta}}=\frac{\beta_{\eta}}{\beta_{\alpha}}
$$

In order to perform a quantitative test of eq 5 we can use the data for the stretching exponents of the segmental relaxation $\beta_{\alpha}$ obtained below. Unfortunately, from our data the corresponding value $\beta_{\eta}$ for the terminal relaxation could not be derived because the plateau zone is not well-defined for the low molecular weight samples used here $\left(M_{\mathrm{w}} \approx 2 \times 10^{4} ; M_{\mathrm{w}} / M_{\mathrm{e}} \approx\right.$ $\left.7-12^{6}\right)$. However, this parameter has been found to lie in the narrow range $\beta_{\eta}=0.55-0.60$ for many monodisperse linear polymers ${ }^{2}$ and we, resultingly, are justified to use the estimate $\beta_{\eta}=0.575 \pm 0.025$ here. The hatched areas in Figure 6 show the ratios to be expected from eq 5 within our estimated error limits.

Table 2 compares the average ratio $R=\log _{10} a_{T, \alpha}$ $\log _{10} a_{T, \eta}$ for each sample to $\beta_{\eta} / \beta_{\alpha}$. Table 2 and Figure 5 show that there are significant differences between the theoretical values and those found in our experiments. However, the predicted trend of increasing thermorheological nonsimplicity with decreasing $\beta_{\alpha}$ is reflected by an increase in $R$, except for one of the samples (PB 70). Considering the crude way of separating segmental and terminal relaxation, the lower values 


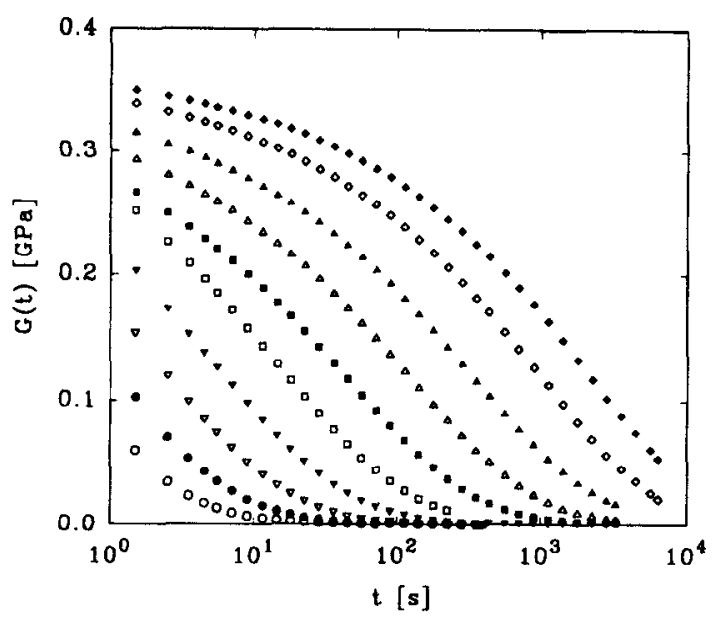

Figure 7. Stress relaxation modulus vs time for polybutadiene sample PB 70. Test temperatures in ${ }^{\circ} \mathrm{C}$ : (O) -39.4; (O) $-40.3 ;(\nabla)-41.2 ;(\nabla)-42.1 ;(\square)-43.0 ;(\mathbb{1})-43.9 ;(\Delta)-45.1$; (৯) $-46.2 ;(\diamond)-47.1 ;(\diamond)-48.1$.

of $R$ from the experiment are not surprising. Any overlap between both relaxations which is not resolved by the splitting leads to an assimilation of the shift factors, i.e. to a decrease of $R$. Furthermore, we note that the use of $\beta_{\eta}=0.515$ instead of the value expected for the terminal dispersion $(\approx 0.55-0.60)$ from the literature ${ }^{2}$ leads to agreement within error bounds except for one of the five samples (PB 70).

It should be noted that in order to obtain timetemperature superposition a systematic variation of the vertical shift factor $b_{T}$ is necessary (Figure 4). Although the determination of $b_{T}$ cannot be done with high accuracy, the variation is significant even if an additional systematic error of about $10 \%$ due to experimental uncertainty such as changes in sample geometry is considered. Similar behavior can be found for all five microstructures. Interestingly, the dip to about $50 \%$ of the high-temperature value is always found near $T_{\mathrm{g}}$, suggesting that this phenomenon may be related to the glass transition.

The variation of $b_{T}$ is also stronger than the factor $T \rho / T_{0} \rho_{0}$ predicted by the Rouse model: ${ }^{6}$ The variation of temperature over the observed range is only $30 \%$; the variation of density can be estimated to $-4.2 \%$ (the negative sign indicating that it counteracts the temperature effect) by assuming the empirical rules of BoyerSpencer ${ }^{22,24}$ and Simha-Boyer ${ }^{23,24}$ for the thermal expansion coefficients in the liquid and glassy states, respectively. The origins of such strong vertical shifting are unclear at this time.

3.2. Stress Relaxation Measurements. Figure 7 shows the stress relaxation moduli of PB 70 at 10 different temperatures, and Figure 8 shows these data reduced to a master curve. There are no signs of deviation from thermorheological simplicity in this instance, which is not surprising because only the segmental relaxation can be observed in this temperature range (near to the glass transition) and in the time scale accessible with the rheometer used in these experiments. The continuous curve in Figure 8 shows a fit with the Kohlrausch-Williams-Watts (KWW) function

$$
G(t)=G_{\mathrm{g}} \exp \left(-(t / \tau)^{\beta_{a}}\right)
$$

where $G_{\mathrm{g}}$ is the limiting value for vanishing time, the
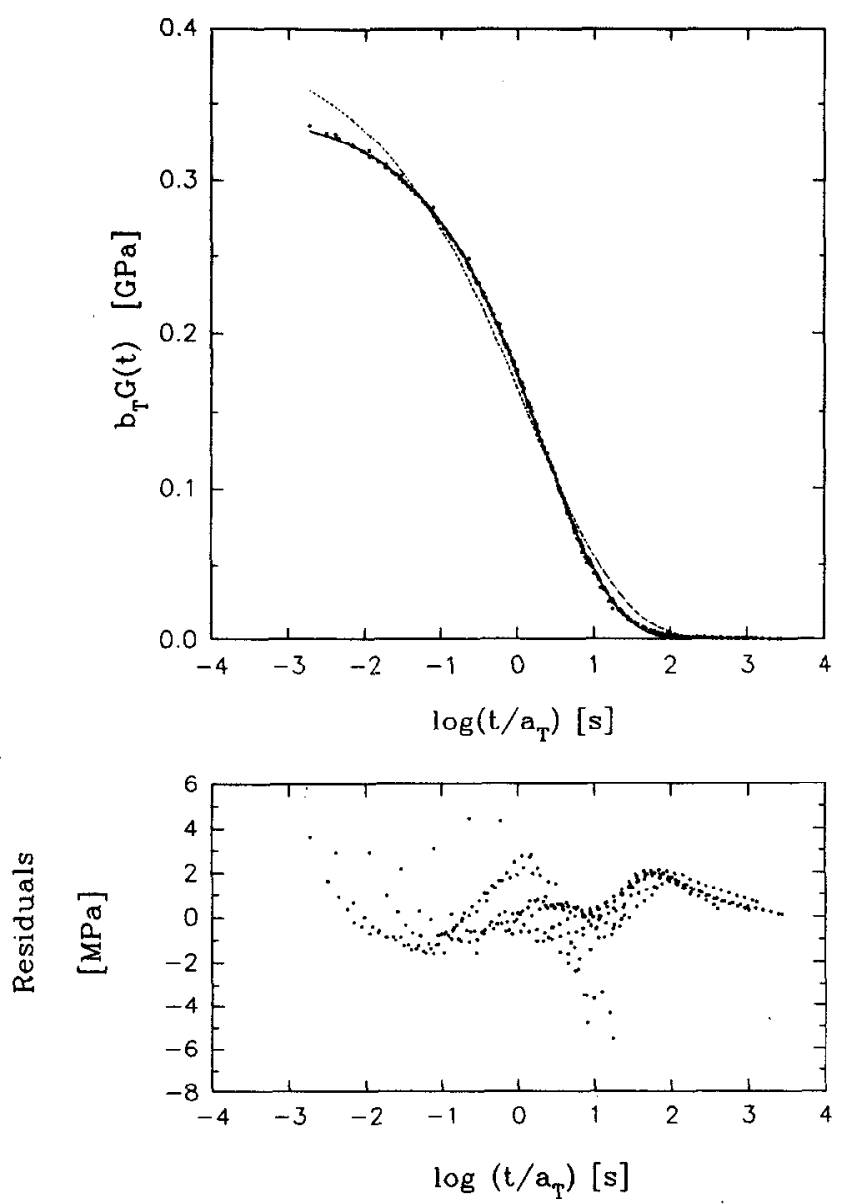

Figure 8. Stress relaxation modulus master curve for polybutadiene sample PB 70 constructed from the data of Figure 7. The continuous line indicates a fit with the $K W W$ expression, eq 6, resulting in the parameters $G_{\mathrm{g}}=(3.44 \pm 0.005) \times$ $10^{8} \mathrm{~Pa}, \beta_{\mathrm{a}}=0.466 \pm 0.001$, and $\tau\left(T=-41.2^{\circ} \mathrm{C}\right)=2.25 \pm 0.01$ s. The dashed line is a fit with $\beta_{\alpha}$ forced to Roland's value $0.35 .^{8}$

glasslike shear modulus, $\beta_{\mathrm{u}}$ is the "stretching exponent" which is a measure of the broadening of the relaxational spectrum, and $\tau$ is the characteristic time of the relaxation (in the case of fitting the whole master curve: its value at the reference temperature). In this expression we omit the addition of the plateau (or entanglement) shear modulus $G_{\mathrm{N}}{ }^{0}$. Because of the low molecular weight of this sample the storage modulus shows no clear plateau; therefore the addition of a constant $G_{\mathrm{N}}{ }^{0}$ is inadequate. ${ }^{35}$ The main result of those fits-that $\beta_{\alpha}$ decreases from 0.49 to 0.41 with increasing vinyl content (see Table 2)-is a decrease of $\beta_{\alpha}$ with increasing vinyl content of the polymer; i.e., the "stretching" of the relaxation increases. In the framework of Ngai's coupling model this would indicate an increase of the cooperativity of the segmental relaxation with an increasing number of vinyl side groups. This is intuitively understandable because those rigid side groups protruding from the backbone should increase the interaction between segments of different polymer chains.

This result was previouly reported by Roland and Ngai. ${ }^{8}$ Although they found the same qualitative dependence of $\beta_{\alpha}$ on the microstructure, the quantitative values of $\beta_{\alpha}$ disagree with ours; the variation is much stronger in their data. This can be clearly seen if one tries to fit, e.g., the stress relaxation modulus data of Figure 8 with the $\beta_{\alpha}=0.35$ from ref 8 . We cannot give a ready explanation for this difference but only indicate that in the cited work the samples were slightly cross- 


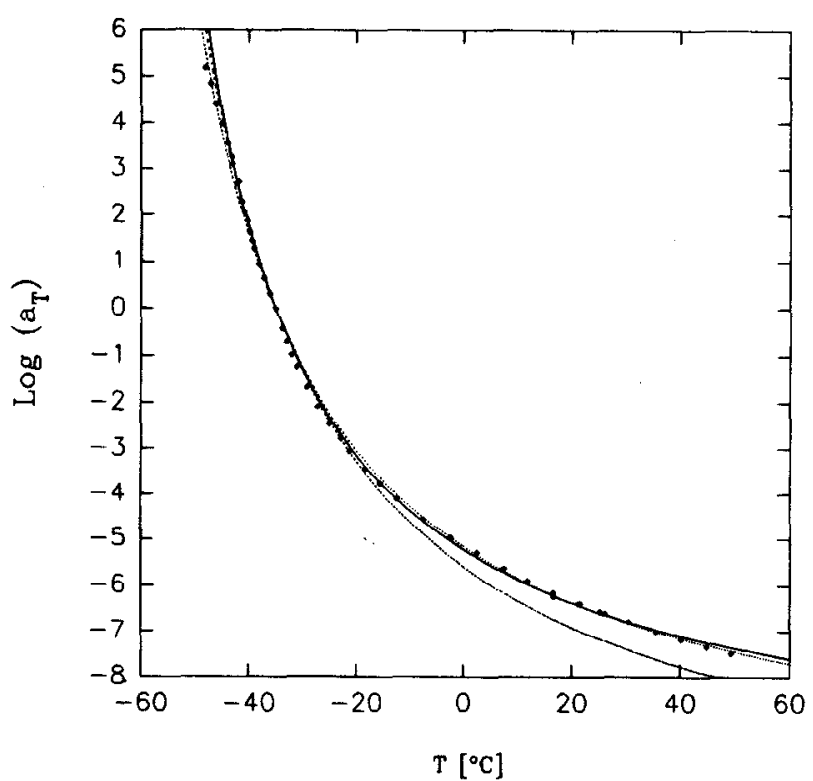

Figure 9. Combined plot of horizontal shift factors from stress relaxation, dynamical measurements, and viscosity for $\mathrm{PB} 70$. The solid curve is a fit with the Williams-Landel-Ferry law for temperatures $\geq-42.1{ }^{\circ} \mathrm{C}$. The short-dashed curve is a fit of the segmental region $\left(-42.1\right.$ to $\left.-35.0^{\circ} \mathrm{C}\right)$ only, the longdashed curve, of the terminal region $\left(-7.5\right.$ to $\left.+49.3^{\circ} \mathrm{C}\right)$ only. The parameters for the fits can be found in Table 3 .

linked in order to attain dimensional stability for uniaxial extension measurements while they were completely un-cross-linked in our case. ${ }^{36}$

3.3. Combined Shift Factors. Assuming thermorheological simplicity, i.e. neglecting the possible differences between segmental and terminal shift factors pointed out in the last section, the results from the different rheological methods can be used to construct the temperature dependence of $a_{T}$ over an extremely large range of 12-14 decades. Figure 9 shows that the values lie on a concave curve when plotted against temperature except for very low temperatures $(T<$ $-42.1^{\circ} \mathrm{C}$ ) where a crossover to a weaker temperature dependence is visible. This feature is found for all five microstructures. It is a quite common phenomenon occurring when cooling below $T_{\mathrm{g}}$ related to the fact that the structure of the glass does not follow the temperature change immediately when it is cooled down. Rather it relaxes slowly into the equilibrium state at temperature $T$. If an experiment is started at a time when this relaxation is not complete, the material will behave as if it were in the equilibrium state at a temperature $T_{\mathrm{f}}>T$, the so-called fictive temperature. Here, this leads to shift factors which are lower than the equilibrium ones. There has been extensive theoretical work on this subject (for an overview see ref 25), but we do not want to deal with it here because the measurements were not done with this intention and would not be sufficient to yield quantitative results.

Except for this region the shift factors can be described by a Williams-Landel-Ferry (WLF) behavior ${ }^{6}$

$$
\log _{10} a_{T}=\frac{-c_{1}^{0}\left(T-T_{0}\right)}{c_{2}^{0}+T-T_{0}}
$$

over the full temperature range with a relative error less than $50 \%$ (parameters in Table 3 ). (Here, $T_{0}$ is the reference temperature of the time-temperature superposition, i.e., $a_{T_{0}}=1$ ), and $c_{1}{ }^{0}$ and $c_{2}{ }^{0}$ are the parameters
Table 3. Parameters for Fits of the WLF Law (Eq 7) to the Combined Rheological Shift Factors Excluding the Low-Temperature Regions Where the Sample Is Not Equilibrated and Their Corresponding VFTH Parameters (Eq 8) ${ }^{a}$

\begin{tabular}{lcccccc}
\hline sample & $\begin{array}{c}T_{0} \\
\left({ }^{\circ} \mathrm{C}\right)\end{array}$ & $c_{1}^{0}$ & $c_{2}^{0}(\mathrm{~K})$ & $T_{\mathrm{VFTH}}\left({ }^{\circ} \mathrm{C}\right)$ & $A(\mathrm{P})$ & $B(\mathrm{~K})$ \\
\hline PB 7 & -95.1 & 11.55 & $29.0[0.9]$ & $-124.1[0.9]$ & $9.8[2.0]$ & $771[25]$ \\
& & {$[0.12]$} & & & & \\
PB 53 & -57.4 & 11.77 & $31.5[0.6]$ & $-88.9[0.6]$ & $3.1[0.5]$ & $854[18]$ \\
& & {$[0.09]$} & & & & \\
PB 70 & -35.0 & 10.34 & $34.2[0.5]$ & $-69.2[0.5]$ & $2.5[0.3]$ & $814[13]$ \\
& & {$[0.08]$} & & & & \\
PB 86 & -23.3 & 12.13 & $22.7[0.7]$ & $-46.0[0.7]$ & $\mathrm{na}^{b}$ & $634[21]$ \\
& & {$[0.16]$} & & & & \\
PB 95 & -0.8 & 11.34 & $28.6[0.8]$ & $-29.4[0.8]$ & $1.5[0.4]$ & $747[23]$
\end{tabular}

a Note that the numbers in brackets are estimates of the asymptotic standard errors of the parameters as determined from the nonlinear curve-fitting procedure (see ref 34 ). ${ }^{b}$ For this sample no viscosity measurements have been done.

to be fitted.) On the large logarithmic scale the error appears small and, considering the experimental errors involved in the rheological measurements, perhaps even tolerable. But a close inspection of the deviations shows that they are of systematic nature.

While the WLF equation is commonly used in the description of the behavior of polymer melts, it is sometimes preferred to use the Vogel-Fulcher-Tamman-Hesse law for the viscosity when dealing with the glass transition event:

$$
\eta_{0}(T)=A \exp \left(\frac{B}{T-T_{\text {VFTH }}}\right)
$$

If one neglects the small temperature dependence of $b_{T}$ in 3, it follows that $a_{T} \propto \eta$. Then the WLF parameters $c_{1}{ }^{0}$ and $c_{2}{ }^{0}$ can be related to the VFTH parameters $A$ and $B$ by

$$
c_{1}^{0}=\frac{1}{\ln 10} \frac{B}{T_{0}-T_{\mathrm{VFTH}}} \quad c_{2}^{0}=T_{0}-T_{\mathrm{VFTH}}
$$

and vice versa by

$$
T_{\mathrm{VFTH}}=T_{0}-c_{2}^{0} \quad B=\ln 10 c_{1}^{0} c_{2}^{0}
$$

The VFTH parameter $A$ cannot be determined from the WLF form because it contains information about the absolute value of the viscosity that is not contained in an expression for the shift factor as in 7 . On the other hand the reference temperature $T_{0}$ in the WLF expression is based on an arbitrary choice and cannot be obtained from the VFTH expression. Hence, the parameters $c_{1}{ }^{0}$ and $c_{2}{ }^{0}$ are not material properties because they depend on the choice of $T_{0}$, but $B$ and $T_{\text {VFTH, in }}$ some sense, are. We, therefore, converted the WLF parameters to VFTH parameters using 10. The prefactor $A$ was determined from the viscosity measurements at high temperatures.

Of course the deviations mentioned above can be due to shortcomings of the WLF/VFTH description because it is mainly derived from phenomenology-only weakly motivated by free volume theories. Several alternative formulas have been proposed which may or may not lead to a better fit. On the other hand, from the preceding discussion of the coupling model it is clear that one has to distinguish between the segmental shift factor, $a_{T, \alpha}$, for low temperatures and the terminal shift factor, $a_{T, \eta}$, 
Table 4. Parameters for the Separate Fits with the WLF Expression for Segmental and Terminal Ranges ${ }^{a}$

\begin{tabular}{lccccccc}
\hline sample & seg range $\left({ }^{\circ} \mathrm{C}\right)$ & $c_{1 \alpha}^{0}$ & term range $\left({ }^{\circ} \mathrm{C}\right)$ & $c_{1 \eta}^{0}$ & $c_{2}^{0}(\mathrm{~K})$ & $c_{1 \alpha}^{0} / c_{1 \eta}^{0}$ & $\beta_{\eta} / \beta_{\alpha}$ \\
\hline PB 7 & $\leq-91.6$ & $18.0[0.7]$ & $\geq-59.6$ & $12.47[0.10]$ & $37.6[0.9]$ & $1.44[0.06]$ & $1.16[0.05]$ \\
PB 59 & $\leq-52.3$ & $15.1[0.6]$ & $\geq-22.4$ & $12.51[0.15]$ & $38.0[1.3]$ & $1.21[0.05]$ & $1.24[0.05]$ \\
PB 70 & $\leq-35.0$ & $11.8[0.5]$ & $\geq-7.5$ & $10.84[0.17]$ & $38.8[1.5]$ & $1.09[0.05]$ & $1.24[0.05]$ \\
PB 86 & $\leq-16.8$ & $16.6[1.2]$ & $\geq 11.6$ & $13.60[0.42]$ & $31.3[2.4]$ & $1.22[0.10]$ & $1.30[0.07]$ \\
PB 95 & $\leq-0.2$ & $14.9[0.6]$ & $\geq 40.4$ & $12.53[0.17]$ & $37.5[1.3]$ & $1.19[0.05]$ & $1.41[0.06]$
\end{tabular}

a The last column gives $\beta_{\eta} / \beta_{\alpha}$ as in Table 2 for comparison. Reference temperatures are chosen as in Table 3. Note that numbers in brackets represent the estimate of the asymptotic standard errors of the parameters as determined from the nonlinear curve-fitting procedure (see ref 34 ).

for high temperatures. So it cannot be expected that a single expression can fit the whole range.

Unfortunately, in the low-temperature range (relative to $T_{\mathrm{g}}$ ) where it can be assumed that the relaxations are virtually purely segmental we have few data due to the onset of nonequilibrium effects. Hence, there is considerable uncertainty in the WLF/NFTH parameters in this range. So a comparison of separate WLF/VFTH laws for the low- and high-temperature ranges suffers from the uncertainty of the fits for the segmental range. ${ }^{37}$ Nevertheless, we attempt an evaluation in the spirit of the coupling model with the additional assumption of WLF behavior for all temperatures. Then, from relation 5 it follows that the separate WLF parameters for segmental and terminal relaxation should fulfill

$$
\frac{c_{1 \alpha}^{0}}{c_{1 \eta}^{0}}=\frac{\beta_{\eta}}{\beta_{\alpha}} \quad c_{2 \alpha}^{0}=c_{2 \eta}^{0}
$$

For the test of this relation we extracted the segmental and terminal range by checking whether the individual moduli curves fall completely into the glass transition or terminal zone of the master curve. Both ranges were fitted with separate $c_{1}{ }^{0}$ but coupled $c_{2}{ }^{0}$. We point out that doing this, we discard the overlap region which is shown in Figure 5 and thus get a result which is independent from the result of Table 2. Table 4 shows the results of these fits for all microstructures. According to what is expected from relation 11 the ratio of the $c_{1}{ }^{0}$ values is always greater than 1 . But there is no quantitative agreement, nor is a clear trend with increasing vinyl content visible, as it was in Table 2.

3.4. Comparison of Different Microstructures: Fragility of Glass Formation. The qualitative success of the Ngai model in explaining the effect of microstructure on the extent of nonrheological simplicity leads to the possibility of using the model, as expanded by Plazek and Ngai, 9 to consider the effect of vinyl content on the "fragility" of these glass-forming systems. The concept of fragility of glass-forming systems was introduced by Angell ${ }^{26,27}$ and provides a useful classification of the relative strength of property changes in going from the liquid state to the glassy state. Strong glass forming liquids change less dramatically in going from the liquid to the glassy state. For this kind of analysis the shift factor (or viscosity or characteristic relaxation time) is plotted logarithmically versus $T_{\mathrm{g}}$ $T .{ }^{28-27}$ If the temperature dependence of the shift factor is given by an Arrhenius law $\exp \left(E_{\mathrm{A}} / k_{\mathrm{B}} T\right)$, this plot yields a straight line, in which case one uses the term "strong" glass formers. Materials in this category are predominantly inorganic network glasses such as $\mathrm{SiO}_{2}$. On the other hand, "fragile" glass formers give lines with curvature indicating that the temperature dependence cannot be described by an Arrhenius law but by

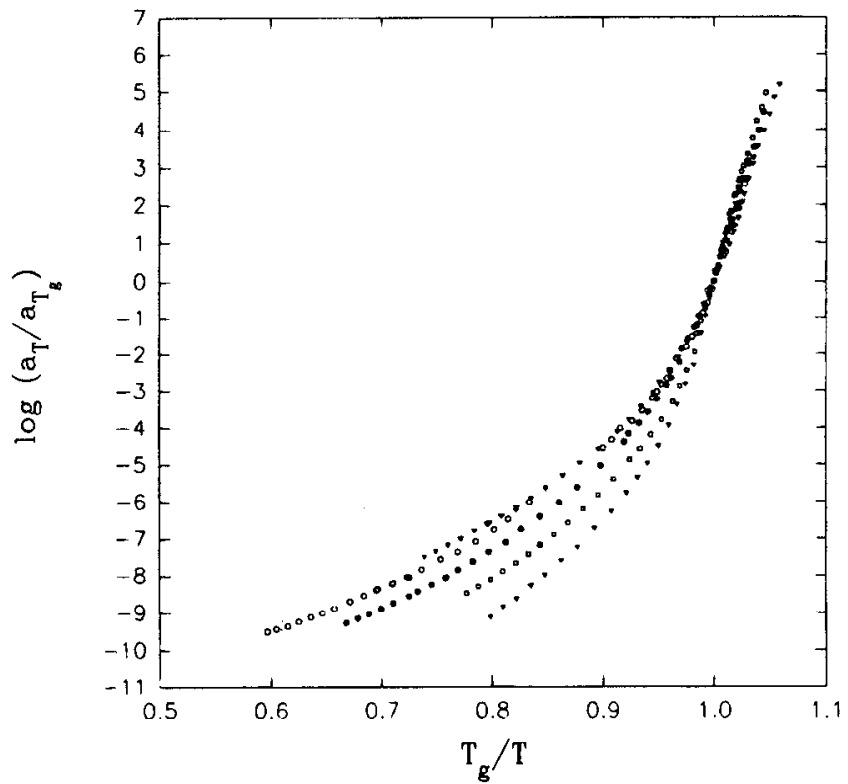

Figure 10. Semilogarithmic plot (Angell plot) of combined shift factors (with reference to the calorimetric glass transition temperature) for all microstructures versus $T_{\mathrm{g}} / T$ where $T_{\mathrm{g}}$ is the calorimetric glass transition temperature too. Symbols: $(O)$ PB 7; (๑) PB 53; ( $)$ PB 70; ( $)$ PB 86; (口) PB 95.

more complex relations such as the Vogel-FulcherTamman-Hesse law used in the last section. Usually, ionic glasses, low molecular weight organic glasses, and glass-forming polymers turn out to be fragile glass formers.

In the case of nonpolymeric materials the glass transition temperature $T_{\mathrm{g}}$ for these plots is conventionally taken as the temperature where the viscosity reaches a value of $10^{12} \mathrm{~Pa} \mathrm{~s}$, which consequently leads to a coincidence of the curves at this point. For polymers this definition is not reasonable because their viscosity is much higher than the viscosity of simple glass formers in a comparable state and furthermore depends strongly on the molecular weight.

At first glance it seems that one could use the glass transition temperature from calorimetric experiments for this purpose since above a certain molecular weight these experiments yield a constant $T_{\mathrm{g}}$. But, as can be seen from Figure 10, this leads to an erratic dependence of the fragility on the vinyl content in the order $70 \%<$ $7 \%<53 \%<95 \%<86 \%$. It appears that the DSC determination of the $T_{\mathrm{g}}$, which includes kinetic phenomena and possible microstructural broadening of the $T_{\mathrm{g}}$ may be inappropriate as a reference point for measurements of molecular dynamics above the $T_{\mathrm{g}}$. Rather, the temperature dependence of the dynamics provides, perhaps, a better reference.

Such an approach for defining the glass transition (or reference) temperature is to set a threshold (usually 10 or $100 \mathrm{~s}$ ) for the Maxwell time $\mathrm{e}^{38}$ which can be calculated 
Table 5. Comparison of Glass Transition Temperatures Obtained from Maxwell Times $t_{M}=10$ and $100 \mathrm{~s}$ and DSC

\begin{tabular}{lccc}
\hline sample & $T_{g}\left(t_{M}=100 \mathrm{~s}\right)\left({ }^{\circ} \mathrm{C}\right)$ & $T_{g}\left(t_{M}=10 \mathrm{~s}\right)\left({ }^{\circ} \mathrm{C}\right)$ & $T_{\mathrm{g}}(\mathrm{DSC})\left({ }^{\circ} \mathrm{C}\right)$ \\
\hline PB 7 & -99.6 & -98.0 & -95 \\
PB 53 & -62.4 & -60.4 & -67 \\
PB 70 & -43.9 & -41.9 & -35 \\
PB 86 & -24.2 & -22.3 & -23 \\
PB 95 & -6.1 & -4.1 & -1
\end{tabular}

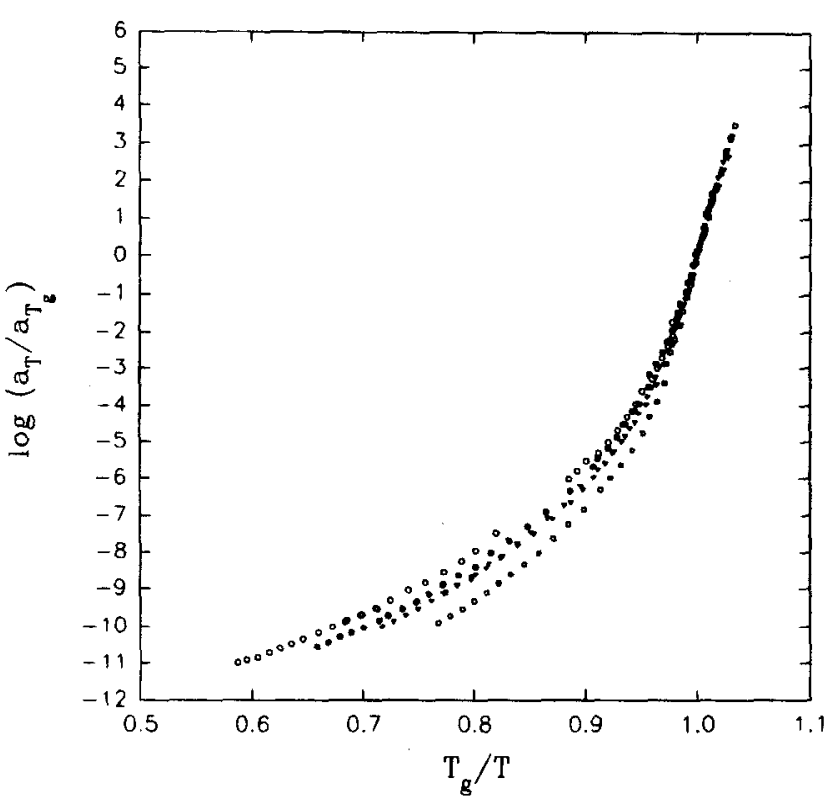

Figure 11. Same plot as Figure 10 but with the glass transition temperature $T_{\mathrm{g}}$ defined as the temperature where the Maxwell time $t_{M}=10 \mathrm{~s}$. Same symbols as Figure 10 .

from the KWW fits as $t_{\mathrm{M}}=(1 / \beta) \Gamma(1 / \beta) \tau_{\mathrm{KWW}}$. Using the WLF expression for the segmental relaxation, one can interpolate the temperature where $t_{M}=10$ and $100 \mathrm{~s}$. Table 5 shows that the $T_{\mathrm{g}}$ values estimated in this way are up to $9 \mathrm{~K}$ higher than those from DSC. When one repeats the "Angell plot" with these choices of $T_{\mathrm{g}}$ instead of the DSC $T_{\mathrm{g}}$ values, a clear tendency of the fragility with microstructure becomes visible: $7 \%<53 \%<70 \%$ $\approx 86 \%<95 \%$ (Figure 11 , the choice $t_{\mathrm{M}}=100 \mathrm{~s}$ yields the same qualitative result).

There have been several proposals for a quantification of the concept of fragility, the first being the ratio

$$
D=B / T_{\mathrm{VFTH}}
$$

often called the "strength parameter" (where $B$ and $T_{\mathrm{VFTH}}$ are the parameters from the VFTH expression). If one assumes strict validity of the VFTH formula and considers the empirical finding that $a_{T \rightarrow \infty} / a_{T \mathrm{~g}} \approx 10^{-16}$, only $D$ determines the shape of the "Angell plot", with smaller $D$ corresponding to higher fragility and $D \rightarrow \infty$ corresponding to the limit of strong glass formers.

The obvious fact that curves in the Angell plot which correspond to more fragile glass formers go through the common point $(1,1)$ more steeply leads to the so-called "steepness index":9,29

$$
S=\left.\frac{\mathrm{d} \log _{10} a_{T}}{\mathrm{~d}\left(T_{\mathrm{g}} J\right)}\right|_{T=T_{\mathrm{g}}}=-\left.T_{\mathrm{g}} \frac{\mathrm{d} \log _{10} a_{T}}{\mathrm{~d} T}\right|_{T=T_{\mathrm{g}}}
$$

$S$ can assume values between 0 and $\infty$ with the higher values corresponding to the more fragile glass formers. It is related to the apparent activation energy at $T_{\mathrm{g}}$ by

$$
S=\frac{1}{\ln 10} \frac{E_{\mathrm{A}}{ }^{\mathrm{app}}\left(T_{\mathrm{g}}\right)}{k_{\mathrm{B}} T_{\mathrm{g}}}
$$

Because the Arrhenius law for strong glass formers can be viewed as the limiting case of VFTH for $T_{\text {VFTH }}=$ 0 , it can also be justified to use the relative position of $T_{\text {VFTH }}$ (with respect to $\left.T_{\mathrm{g}}\right)^{30}$

$$
F=\frac{T_{\mathrm{g}}-T_{\mathrm{VFTH}}}{T_{\mathrm{g}}}
$$

as a measure of fragility. Here, $F=1$ denotes the "strong limit" $F \rightarrow 0$ indicates increasing fragility.

Expressions $13(S)$ and $15\left(F^{\prime}\right)$ make use of the glass transition temperature and are therefore affected by the uncertainty of its definition. Only expression $12(D)$ is independent of $T_{\mathrm{g}}$, but it supposes the validity of VFTH. As shown in the previous section, VFTH parameters depend strongly on the region of fit and so does $D$. So all the definitions are subject to a certain arbitrariness and it is not a priori clear that they result in the same classification of glass formation strength.

Table 6 shows some of the possible variants of calculating "fragility measures". The parameter $D$ derived from VFTH fits of the whole data set results in a fragility order $7 \%<53 \%<70 \%<95 \%<86 \%$. Although this result is unambiguous, considering the small error bars of $D$, it should be questioned after the above the discussion of different shift factors for the terminal and segmental regions. If only the segmental range defined in the preceding section is taken into account the values of $D$ change to about twice their values, yielding the order $7 \%<53 \%<86 \% \approx 95 \%<$ $70 \%$. Due to the smaller number of data the errors increase here so that a clear distinction of fragility between PB 70, PB 86, and PB 95 is not possible from this analysis. Calculating $S$ at $T_{\mathrm{g}}$ from DSC measurements yields a similar erratic behavior, as in Figure 10, while using $T_{\mathrm{g}}$ defined by $t_{\mathrm{M}}=10 \mathrm{~s}$ gives $7 \%<53 \%<$ $70 \%<86 \%<95 \%$, but the three lower values cannot be distinguished with certainty. Finally, the calculation of $F$ results in $7 \%<53 \%<70 \%<86 \%=95 \%$ or $7 \%<$ $53 \%<70 \%<86 \%<95 \%$, dependent on the choice of the fit region for $T_{\mathrm{V} \text { FTH. }}$. Again, considering the argument of different shift factors, the second variant using only the segmental region has to be preferred.

Despite the uncertainty resulting from the conceptual difficulties the most reasonable order of fragility seems to be a monotonic increase with vinyl content. This is also what would be expected from the coupling model ${ }^{9}$ because an increase of the coupling parameter $1-\beta_{\mathrm{a}}$ should give rise to stronger deviations from the Arrhenius law. But this model allows an even more comprehensive statement: The "fundamental" shift factor

$$
a_{\mathrm{T}}^{\circ}=a_{T, \alpha}^{\beta_{a}}
$$

should show the same temperature dependence with respect to $\left(T-T_{\mathrm{g}}\right) / T_{\mathrm{g}}$ for different polymers. Figure 12 shows the fundamental shift factor for the five polybutadienes in the segmental range. Indeed, the values fall on one curve. However, since the differences in $\beta_{\alpha}$ are not very large, this is a weak argument in favor of the theory. It is remarkable that this correspondence also extends to the "nonequilibrium" region for $T \leq$ $0.98 T_{\mathrm{g}}$.

\section{Summary}

A study of the influence of the microstructure (vinyl content) on the chain dynamics in the terminal and 
the National Institute of Standards and Technology for its support during his stay as a Foreign Guest Scientist in the Polymers Division. We are grateful to $\mathbf{M r}$. M. Zähres (Universität Duisburg) for the NMR measurements and to Dr. J. Weese (Freiburger Materialforschungszentrum) for providing a copy of LSSHIFT and developing a PC version of the program. We also thank Mr. Bill Roberts of the Building and Fire Research Laboratory at NIST for access to the modulated DSC. We thank Dr. K. L. Ngai for valuable discussions and providing a preprint of ref 3 .

Note added in proof: The reviewers of the manuscript suggested that the original data be obtainable in digital form from the authors upon request. ASCII data files have been stored at both NIST and the Forschungzentrum Jülich and they can be obtained from either location by request from either R. Zorn (Jülich) or G.B. McKenna (NIST).

\section{References and Notes}

(1) Ngai, K. L.; Plazek, D. J. J. Polym. Sci., Part B: Polym. Phys. $1986,24,619-632$.

(2) Plazek, D. J.; Zheng, X. D.; Ngai, K. L. Macromolecules 1992 , 25. 4920-4924.

(3) Ngai, K. L. Universal Patterns of Relaxations in Complex Systems, to be published in Disorder Effects on Relaxation Processes; Richert, R., Blumen, A., Eds.; Springer: Berlin.

(4) Morton, M.; Fetters, L. J. Rubber Rev. 1975, 48, 359.

(5) Bahary, W. S.; Sapper, D. I.; Lane, J. H. Rubber Chem. Technol. 1967, 40, 1529-1543.

(6) Ferry, J. D. Viscoelastic Properties of Polymers; John Wiley \& Sons: New York, 1980.

(7) Carella, J. M.; Graessley, W. W.; Fetters, L. J. Macromolecules 1984, 17, 27752786.

(8) Roland, C. M.; Ngai, K. L. Macromolecules 1991, 24, 53155319.

(9) Plazek, D. J.; Ngai, K. L. Macromolecules 1991, 24, 12221224.

(10) Van der Velden, G.; Didden, C.; Veermanns, T.; Beulen, J. Macromolecules 1987, 20, 1252.

(11) Sato, H.; Takebayashi, K.; Tanaka, Y. Macromolecules 1987, $20,2418$.

(12) Bywater, S. Polym. Commun. 1983, 24, 203.

(13) Bywater, S.; Firat, Y.; Black, P. E. J. Polym. Sci., Polym Chem. Ed. 1984, 22, 669 .

(14) Halasa, A. F.; Schulz, D. N.; Tate, D. P.; Mockel, V. D. Adv. Organomet. Chem. 1980, $18,55$.

(15) Halasa, A. F.; Lohr, D. F.; Hall, J. E. J. Polym. Sci., Polym. Chem. Ed. 1981, 19, 1357.

(16) Colby, R. H.; Fetters, L. J.; Graessley, W. W. Macromolecules 1987, 20, 2226-2237.
(17) Ni, S.; Shen, L.; Yu, F.; Qian, B. J. Appl. Polym. Sci. 1990, $41,13-23$.

(18) Di Marzio, E. A.; Gibbs, J. H. J. Polym. Sci. 1959, 40, 121131.

(19) Roland, C. M. To be published in Macromolecules.

(20) Honerkamp, J.; Weese, J. Rheol. Acta 1998, 32, 57-64.

(21) Plazek, D. J. J. Phys. Chem. 1965, 69, 3480-3487.

(22) Boyer, R. F.; Spencer, R. S. J. Appl. Phys. 1944, 15, 398.

(23) Simha, R.; Boyer, R. F. J. Chem. Phys. 1962, 37, $1003-1007$.

(24) van Krevelen, D. W.; Hoftyzer, P. J. Properties of Polymers; Elsevier: Amsterdam, 1976.

(25) McKenna, G. B. Glass Formation and Glassy Behavior. In Polymer Properties; Booth, C., Price, C., Eds.; Comprehensive Polymer Science, Vol. 2; Pergamon: Oxford, U.K. 1989; pp $311-362$.

(26) Angell, C. A.; Sichina, W. Ann. N.Y. Acad. Sci. 1967, 279, 53-67.

(27) Angell, C. A. J. Phys. Chem. Solids 1988, 49, 863-871

(28) Laughlin, W. T.; Uhlmann, D. R. J. Phys. Chem. 1972, 76, $2317-2325$

(29) Böhmer, R.; Angell, C. A. Phys. Rev. B 1992, 45, 1009110094.

(30) Hodge, I. M. Macromolecules 1987, 20, 2897-2908.

(31) Böhmer, R.; Ngai, K. L.; Angell, C. A.; Plazek, D. J. J. Chem. Phys. 1993, 99, 4201-4209.

(32) Certain commercial materials and equipment are identified in this paper to specify adequately the experimental procedure. In no instance does such identification imply recommendation or endorsement by the National Institute of Standards and Technology, nor does it necessarily imply that the product is the best available for the purpose.

(33) Douglas, J. F.; Hubbard, J. B. Macromolecules 1991, 24, 3163-3177.

(34) SigmaPlot Scientific Graphing Software. Jandel Scientific: San Rafael, CA, 1992.

(35) Anyway, an introduction of this term changes the fit only slightly; e.g. the values of $\beta_{\alpha}$ would increase by only $0.002-$ 0.012 .

(36) In a paper not yet published, Roland ${ }^{19}$ finds $\beta_{a}=0.41$ for un-cross-linked 1,2-polybutadiene identical with our value and a decrease of $\beta_{\alpha}$ with increasing cross-link density supporting our presumption that these differences are due to their cross-linking of the polybutadiene.

(37) At this point a cautious error analysis is important because the WLF parameters are strongly interrelated during the fit. It is easy to get an apparently good fit from this range, too, but calculation of the errors leads to relative uncertainties of $c_{1}{ }^{0}$ which are 6-8 times higher than for the full temperature range. (This remark may be understood as a general warning when one compares literature results on VFTH or WLF parameters from small data sets too.)

(38) This procedure may seem less elegant because of the arbitrariness of the choice of this threshold, but the $T_{\mathrm{g}}$ from DSC involves a choice of heating rate which influences the obtained value in a similar way.

MA9502330 